\title{
Development and validation of a multivariable prediction model of spontaneous preterm delivery and microbial invasion of the amniotic cavity in women with preterm labor
}

\author{
Teresa Cobo, MD, PhD; Victoria Aldecoa, MD; Francesc Figueras, MD, PhD; Ana Herranz, MD, PhD; Silvia Ferrero, MD, PhD; \\ Montse Izquierdo, MD; Clara Murillo, MD; Raquel Amoedo, MD; Claudia Rueda, MD; Jordi Bosch, MD; PhD; \\ Raigam J. Martínez-Portilla, MD; Eduard Gratacós, MD, PhD ${ }^{1}$; Montse Palacio, MD, PhD ${ }^{1}$
}

BACKGROUND: Early spontaneous preterm delivery is often associated with microbial invasion of the amniotic cavity and/or intraamniotic inflammation.

OBJECTIVE: The objective of the study was to develop and validate clinically feasible multivariable prediction models of spontaneous delivery within 7 days and microbial invasion of the amniotic cavity in women admitted with diagnose of preterm labor and intact membranes below 34 weeks.

STUDY DESIGN: We used data from a cohort of women admitted from 2012 to 2018 with diagnosis of preterm labor below 34 weeks who had undergone amniocentesis to rule out microbial invasion of the amniotic cavity. The main outcome was spontaneous delivery within 7 days from admission. The secondary outcome was microbial invasion of the amniotic cavity, defined by a positive culture and/or 16S ribosomal RNA gene in the amniotic fluid. The sample $(n=358)$ was divided into derivation (2012-2016) and validation cohorts (2017-2018). Logistic regression models using a stepwise selection of variables were developed for the outcomes evaluated. We explored as predictive variables ultrasound cervical length measurement at admission, maternal C-reactive protein, gestational age, amniotic fluid glucose, and interleukin-6 (expressed as log units). Models were developed in the derivation cohort and applied to the validation cohort and diagnostic performance was calculated.

RESULTS: The derivation cohort included 263 women and the validation cohort 95 women. One hundred five of the women (39\%, 105 of 268) spontaneously delivered in the following 7 days and 68 (19\%, 68 of 358) had microbial invasion of the amniotic cavity. For spontaneous delivery within 7 days after admission, 4 predictors were identified: cervical length at admission, gestational age, amniotic fluid glucose, and interleukin-6. The diagnostic performance of the model was assessed in the validation cohort using the receiver operating characteristic curve and showed an area under curve of 0.86 (95\% confidence interval, $0.77-0.95)$ with a detection rate of spontaneous delivery within 7 days of $87 \%$, a falsepositive rate of $33 \%$, a negative predictive value of $80 \%$, and a negative likelihood ratio of 0.1908 . For microbial invasion of the amniotic cavity, 2 independent predictors of the amniotic cavity were identified: amniotic fluid glucose and maternal C-reactive protein. The receiver operating characteristic curve and an area under curve in the validation cohort was 0.83 (95\% confidence interval, $0.70-0.96)$ with a detection rate of $76 \%$, a false-positive rate of $8 \%$, a negative predictive value of $93 \%$, and a negative likelihood ratio of 0.2591 .

CONCLUSION: In women with preterm labor, we propose 2 clinically feasible prediction models to classify as low vs high risk of spontaneous delivery within 7 days and of microbial invasion of the amniotic cavity. The models showed a high diagnostic performance and could be of value to optimize clinical management.

Key words: amniocentesis, cervical length, interleukin-6, intraamniotic infection, intraamniotic inflammation, microbial invasion of the amniotic cavity, multivariable prediction models, preterm birth, preterm labor, spontaneous preterm delivery
$\mathrm{O}$ ne third of women who are admitted to hospitals with a diagnosis of preterm labor and intact membranes (PTL) below 34 weeks will deliver within the following 7 days. ${ }^{1,2}$ These cases are more often associated with microbial invasion of the amniotic

Cite this article as: Cobo Teresa, Aldecoa V, Figueras F, et al. Development and validation of a multivariable prediction model of spontaneous preterm delivery and microbial invasion of the amniotic cavity in women with preterm labor. Am J Obstet Gynecol 2020; Xxx; xx-xx

$0002-9378 / \$ 36.00$

(c) 2020 Elsevier Inc. All rights reserved.

https://doi.org/10.1016/j.ajog.2020.02.049 cavity (MIAC) and/or intraamniotic inflammation (IAI). ${ }^{2-4}$

Identification of women with PTL at high risk of delivery within 7 days and/or of MIAC remains an unsolved clinical challenge. Several ultrasound (US) ${ }^{5}$ and biochemical markers ${ }^{6}$ have been proposed as predictors of spontaneous preterm delivery within 7 days. ${ }^{7}$ Similarly, amniotic fluid glucose ${ }^{8-12}$ or interleukin (IL)-6 concentrations ${ }^{13-15}$ have been strongly related to MIAC and/or IAI. However, none of these markers have shown enough accuracy to be used as stand-alone predictors in clinical practice.

There is growing evidence suggesting that multivariable prediction models can improve the diagnostic performance of different adverse outcomes such as preeclampsia ${ }^{16}$ or fetal growth restriction. ${ }^{6}$ Concerning spontaneous preterm delivery, different prediction models have been proposed. In women with PTL, Carter et $\mathrm{al}^{17}$ developed and validated a multivariable prediction model (QUiPP app) that integrates maternal risk factors (symptoms, previous cervical surgery, previous preterm birth $<37.0$ weeks, previous preterm prelabor rupture of membranes, number of fetuses), US (transvaginal ultrasound assessment of cervical length), and biochemical markers (cervicovaginal fluid quantitative fetal fibronectin test results) to 


\section{AJOG at a Glance}

\section{Why was the study conducted?}

To develop feasible multivariable prediction models of spontaneous preterm delivery within 7 days and microbial invasion of the amniotic cavity in women with preterm labor

\section{Key findings}

The model to predict spontaneous preterm delivery within 7 days included gestational age at admission, ultrasound cervical length measurement, amniotic fluid glucose, and inteleukin- 6 and showed an area under the curve of 0.86 (95\% confidence interval, 0.77-0.95) with a detection rate of spontaneous delivery within 7 days of $87 \%$ and a false-positive rate of $33 \%$. The model to predict microbial invasion of the amniotic cavity included maternal C-reactive protein and amniotic fluid glucose and showed an area under the curve of 0.83 (95\% confidence interval, $0.70-0.96$ ) with a detection rate of $76 \%$ and a false-positive rate of $8 \%$.

\section{What does this add to what is known?}

The good diagnostic performance observed in these models might encourage clinicians to integrate the use of the amniocentesis, particularly in women with early preterm labor, to efficiently target the high-risk group of spontaneous delivery within 7 days and microbial invasion of the amniotic cavity, avoiding unnecessary overtreatment if the risk is low.

predict the occurrence of spontaneous preterm delivery. ${ }^{17}$ However, the model was not designed to predict IAI and/or MIAC.

In this regard, other authors have proposed different combinations of proteomic biomarkers in the amniotic fluid and in the cervicovaginal fluid ${ }^{18-20}$ with a good accuracy to predict MIAC and/or IAI. These models have not been properly validated ${ }^{20}$ or are not feasible to be used as a tool for a rapid diagnosis in the clinical setting. ${ }^{18,19}$

In this scenario, we aimed to develop and validate clinically feasible multivariable prediction models of both spontaneous delivery within 7 days after admission and MIAC in women with PTL below 34 weeks of gestation that can be used in clinical decision making.

\section{Material and Methods}

\section{Patient population}

This retrospective, observational study included consecutive women recruited within a common research line for the prediction of adverse outcomes in PTL from 2012 to 2018 at the Hospital Clinic and Hospital Sant Joan de Déu, Barcelona. As part of institutional clinical protocols, women with singleton pregnancies admitted with diagnosis of preterm labor and intact membranes below 34 weeks were offered an amniocentesis.

Women with the following conditions were excluded: clinical signs of chorioamnionitis ${ }^{21}$ at admission, cervical length measurement at admission greater than the fifth centile, ${ }^{2}$ maternal age $<18$ years, and no consent to perform amniocentesis for this indication. Maternal characteristics of women who declined amniocentesis were similar to our study population.

Patient selection and sampling procedures of both studies were performed in accordance with the Declaration of Helsinki and applicable local regulatory requirements after approval from the Institutional Review Boards (HCB/2010/ 5811, HCB/2015/0367, PIC-82-15). Written informed consent was obtained for sample collection from all subjects.

The study group was divided into a derivation cohort and a validation cohort.

\section{Clinical management}

Standard management of women with a diagnosis of PTL included US transvaginal cervical length measurement and maternal blood analysis for evaluation of maternal C-reactive protein (CRP) and white blood cell count at admission. Transvaginal cervical length was measured by experienced staff following Fetal Medicine Foundation guidelines (htts://www.fetalmedicine. com). Briefly, the vaginal probe was placed approximately $3 \mathrm{~cm}$ from the cervix to avoid pressure resulting in distortion of the position and shape of the cervix. A sagittal view of the full length was measured by placing the calipers at the farthest points at which the cervical walls were juxtaposed. Ultrasound cervical length was measured at least 3 times and the shortest measurement was recorded.

A complete course of antenatal steroids, betamethasone $12 \mathrm{mg}$ intramuscular injection with 2 doses given 24 hours apart, was administered until $34+6$ weeks for fetal lung maturation. If there was no clinical contraindication, tocolysis (nifedipine or atosiban) was administered to prolong pregnancy during steroid administration (a course of 48 hours).

Broad-spectrum antibiotics (endovenous ampicillin $1 \mathrm{~g}$ every 6 hours and gentamycin $80 \mathrm{mg}$ every 8 hours and 1 dose of oral azithromycin $1 \mathrm{~g}$ ) were administered in women with amniotic fluid glucose concentrations $<5 \mathrm{mg} / \mathrm{dL}$ and/or with microorganisms identified by amniotic fluid Gram staining and/or positive amniotic fluid cultures.

In women with advanced cervical dilatation (Bishop >6), we also started prophylactic broad-spectrum antibiotics that were discontinued if amniotic fluid cultures were negative. In the case of the onset of uterine contractions after these 48 hours of steroid administration, tocolysis was reintroduced only if MIAC or clinical chorioamnionitis were excluded.

Cultures for genital mycoplasma (Mycoplasma IST 2, bioMérieux for Ureaplasma spp or Mycoplasma hominis), aerobic (chocolate agar), and anaerobic (Schaedler agar for anaerobes and thioglycollate broth) bacteria as well as amniotic fluid glucose concentrations and Gram stains were performed 
immediately after amniocentesis and clinical management was made according to the results.

Amniotic fluid samples were also analyzed by specific polymerase chain reaction (PCR) amplification of the $16 \mathrm{~S}$ ribosomal RNA gene using the primers: 5-AGA GTT TGA TCC TGG CTC AG3and 5-GGA CTA CCA GGG TAT CTA AT-3 followed by Sanger sequencing in the Department of Microbiology. Sequences were identified using the Blast algorithm in the National Center for Biotechnology Information database, with a minimum $98 \%$ sequence identity.

Amniotic fluid IL-6 concentrations were measured by enzyme-linked immunoassay (Biosource; Invitrogen, Carlsbad, CA) in amniotic fluid samples previously centrifuged at $4000 \mathrm{rpm}$ for 10 minutes at $4{ }^{\circ} \mathrm{C}$ and stored at $-80^{\circ} \mathrm{C}$. The minimum detectable level of IL- 6 was $0.2 \mathrm{ng} / \mathrm{mL}$.

Information of $16 \mathrm{~s}$ ribosomal RNA gene sequencing and IL-6 was not available for clinical decision making.

\section{Classification of outcomes}

The primary outcome was spontaneous delivery within 7 days after admission. Women who delivered because of maternal or fetal indications were consequently censored. The secondary outcome was the occurrence of MIAC, defined by the presence of a positive amniotic fluid culture for bacteria, fungi, and Ureaplasma spp or Mycoplasma hominis and/or by specific PCR amplification of the 16S ribosomal RNA gene. Gestational age was established according to crown-rump length at the firsttrimester US scan. ${ }^{22}$

\section{Predictors}

Predictors used to develop multivariable prediction model were those that showed to be independent predictors in the univariate logistic regression analysis and included the following continuous variables: US cervical length measurement (millimeters) at admission, maternal CRP concentrations (milligrams per liter), gestational age (weeks), amniotic fluid glucose concentrations (milligrams per deciliter), and amniotic fluid IL-6 (nanograms per milliliter) (expressed in a log scale).

\section{Sample size}

The sample size $e^{23}$ to develop the multivariable prediction model was established assuming an initial inclusion of 5 potential predictors selected a priori based on consensus among the investigators (T.C., M.P., F.F., E.G.) and for an outcome (delivery within 7 days after admission) with a prevalence of $30 \%,{ }^{1,2}$ resulting in a sample size of 167 . To account for a proportion of censored cases in which delivery is indicated electively (30\%) and $10 \%$ of cases with missing information for any of the predictors, a conservative number of 240 cases were estimated.

For the validation cohort, all consecutive cases attended within 2 years (2017-2018) after the model was developed were included.

\section{Statistical analysis}

Statistical analysis was performed using SPSS 20.0 for MAC OS (IBM Corporation, Armonk, NY). We compared maternal characteristics and perinatal outcomes between the derivation and the validation cohorts; continuous variables were compared using a nonparametric Mann-Whitney $U$ test presented as median with interquartile range $\left(25^{\text {th }}\right.$, 75th percentile). Categorical variables were compared using the $\chi^{2}$ or Fisher exact test. Differences were considered statistically significant with a value of $P$ $<.05$ with 2 -sided alternative hypotheses.

Multivariable analysis by stepwise logistic regression was used to identify independent factors associated with the outcomes (prediction model development). The models that could best predict spontaneous delivery within 7 days and MIAC were constructed based on the final regression model and the direction of effects. Goodness-of-fit models were assessed by calculating Nagelkerke's $R^{2}$.

Diagnostic performance was calculated (area under the receiver-operating characteristic (AUROC) curve, sensitivity, specificity, positive predictive value, negative predictive value, positive likelihood ratio, and negative likelihood ratio for spontaneous delivery within 7 days and for MIAC in the derivation and the validation cohorts.

A posterior probabilities for a given outcome were calculated using Fagan's plots ( $\mathrm{R}$ statistics [ $\mathrm{R}$ project; package ggplot2]), in which a priori risk was based on the prevalence of the outcome among the sample population multiplied by the positive and negative likelihoods ratios of the diagnostic test.

Finally, to assess the likelihood of patients to have significantly different results if receiving tocolysis based on their clinical characteristics, we performed a nearest-neighbor propensity score matching by logit regression, in which the outcome variable was spontaneous delivery within 7 days after admission. Predictor variables were gestational age at admission, cervical length, amniotic fluid glucose, and IL-6, and the treatment variable was tocolysis. Test balance for adequate matching was performed using variance ratios.

\section{Results}

During the study period 531 women were eligible for the study and 358 were finally included. Figure 1 shows flow chart of the entire study population.

For the overall population $(\mathrm{n}=358)$, 105 (39\%, 105 of 268) of women spontaneously delivered in the following 7 days and $68(19 \%, 68$ of 358) had microbial invasion of the amniotic cavity. Microorganisms isolated in the amniotic fluid with their amniotic fluid IL-6 concentrations are presented in a supplemental table (Supplemental Table 1).

The majority of women with MIAC (60 of $67,89.6 \%$ ) had high levels of amniotic fluid IL-6 $(\geq 2.6 \mathrm{ng} / \mathrm{mL})$. Women with MIAC had an earlier gestational age at admission (median [25th, 75th percentiles], 26.4 [24.2, 30.5] weeks) than women without (28.6 [25.6, 30.9] weeks). Gestational age at delivery was significantly earlier in women with MIAC (27 [25.1, 31.1] weeks) than in women without (35.3 [30.3, 38.5] weeks), and latency to delivery was significantly shorter in women with $\operatorname{MIAC}(1[0,3]$ day vs $35[6,64.5]$ days, respectively). 


\section{FIGURE 1}

Distribution of the entire study group (2012-2018)

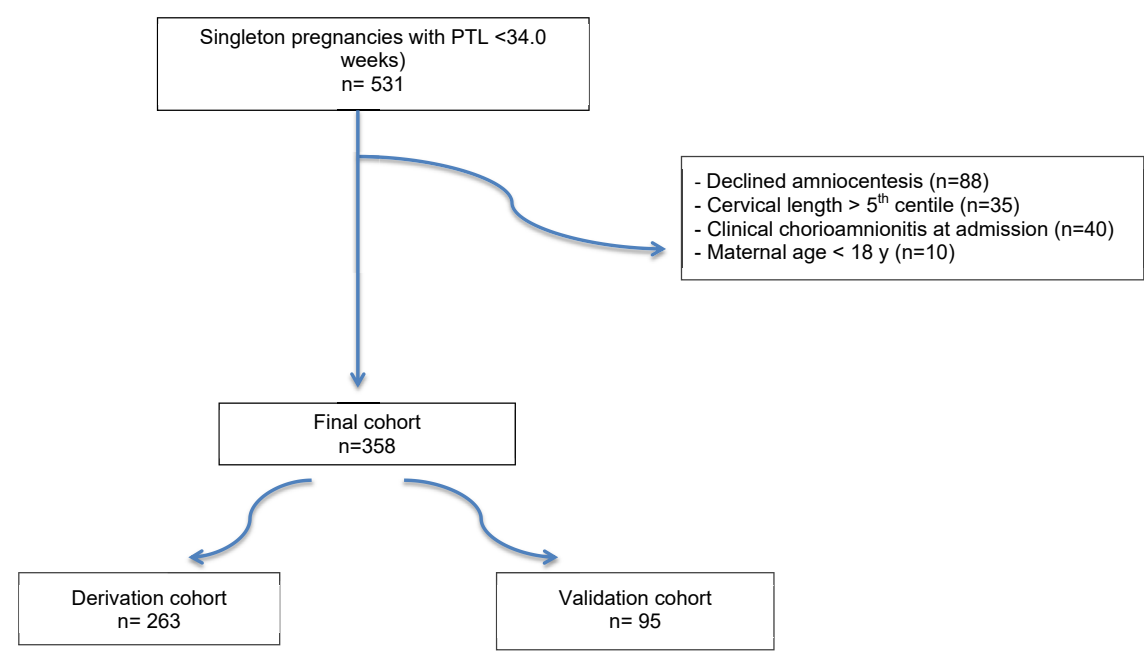

Cobo et al. Prediction models of spontaneous preterm delivery and microbial invasion of the amniotic cavity in preterm labor. Am J Obstet Gynecol 2020.

Among women admitted below 28 weeks of gestation, $48 \%$ (59 of 123) spontaneously delivered in the following 7 days, and $24.6 \%$ (41 of 167) had MIAC. Below 32 weeks of admission, the prevalence of spontaneous delivery within 7 days and MIAC was $38.7 \%$ (86 of 222) and $19.1 \%$ (58 of 304 ), respectively.

Maternal characteristics and perinatal outcome comparisons between women with and without MIAC and with and without SPTD within 7 days are presented in supplemental tables (Supplemental Tables 2 and 3, respectively).

The derivation cohort included 263 women and the validation cohort 95 . Differences in the maternal characteristics and perinatal outcomes between women from the derivation and the validation cohorts are shown in Table 1. In the validation cohort, IL- 6 concentrations were significantly higher, gestational age at delivery was significantly earlier and latency to delivery significantly was shorter than in the derivation cohort.

Multivariable analysis indicated that gestational age at admission, cervical length, amniotic fluid glucose, and IL-6 were independent predictors for spontaneous delivery within 7 days (Table 2). Maternal CRP and amniotic fluid glucose were independent predictors of MIAC $(P<.05)$.

The regression formula for spontaneous delivery within 7 days was: $-7.588+0.132$ * gestational age at admission $-0.051 *$ US cervical length $0.055^{*}$ amniotic fluid glucose +1.438 * amniotic fluid $\log$ (IL-6). $\mathrm{R}^{2}=51.5 \%$.

The regression formula for MIAC was $=1.034+0.169 *$ maternal CRP $-0.158 *$ amniotic fluid glucose. $\mathrm{R}^{2}=$ $65.6 \%$.

According to the selected predictors and the direction of effects, we proposed 2 models: one for spontaneous delivery within 7 days and one for MIAC in which maternal CRP = milligrams per liter; amniotic fluid glucose $=$ milligrams per deciliter; gestational age at admission = weeks; US cervical length = millimeters; and amniotic fluid IL-6 (nanograms per milliliter) were expressed in a $\log$ scale.

The diagnostic performance of the model for predicting spontaneous delivery within 7 days and MIAC was assessed using ROC curves. The AUROC of the model to predict the risk of spontaneous delivery within 7 days was $0.88(95 \%$ confidence interval [CI], $0.83-0.93)$ in the derivation cohort and $0.86(95 \%$ CI, $0.77-0.95)$ in the validation cohort. In the validation cohort, the detection rate of spontaneous delivery within 7 days was of $87 \%$ and the false-positive rate was $33 \%$. We selected a cutoff with a high detection rate to efficiently target the high-risk group of women who will inevitably deliver in the following days.

The AUROC of the model for predicting MIAC was $0.94 \quad(95 \% \mathrm{CI}$, $0.89-0.98)$ in the derivation and 0.83 (95\% CI, 0.70-0.96) in the validation cohort. In the validation cohort, the detection rate of MIAC was $76 \%$ of women, and the false-positive rate was of $8 \%$. We decided to select a cutoff with a low false-positive rate to avoid unnecessary antibiotic treatment in women without MIAC. The diagnostic performance of the 2 models is shown in Table 3.

Figure 2 shows Fagan nomogram of the validation cohort. According to this, if we test the model for spontaneous delivery within 7 days in a woman with a priori risk of $57 \%$ this risk increases to $79 \%$ in the high-risk group (after applying the positive LR value) and decreases to $20 \%$ in the low-risk group (after applying the negative LR). Regarding the risk of MIAC, if the priory risk is $22 \%$, it increases to $73 \%$ in the high-risk group and decreases to $7 \%$ in the low-risk group.

Our analysis (propensity score) to account for an indication bias suggests a lack of such effect because we did not find tocolysis to be associated with spontaneous delivery within 7 days (Supplemental Table 4).

\section{Comment}

\section{Principal findings}

In this study, we developed and validated clinically feasible multivariable prediction models in women admitted with a diagnosis of PTL below 34 weeks of gestation that could help clinicians to efficiently manage low- and high-risk populations of spontaneous delivery within 7 days and of MIAC.

The good diagnostic performance observed in these models might encourage clinicians to integrate the use of the amniocentesis, considered a safe procedure, even in a more challenging 


\section{TABLE 1}

Maternal characteristics and perinatal outcomes of the women in the derivation and the validation cohorts

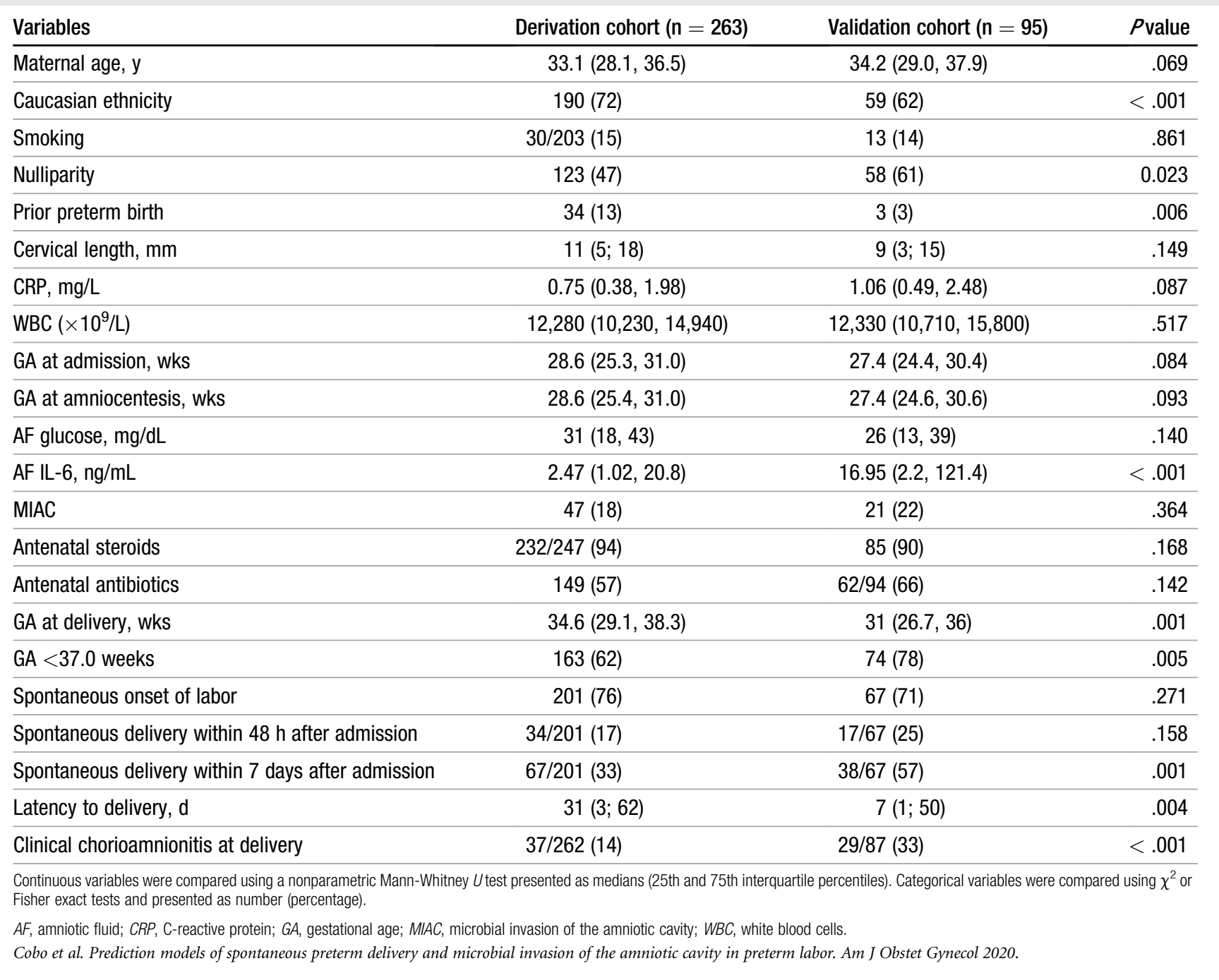

condition such as preterm prelabor rupture of membranes, ${ }^{24}$ as part of the management of PTL, particularly in women with early PTL (eg, before 32 weeks).

\section{Results}

Prediction models of spontaneous delivery within 7 days after admission have previously been proposed in symptomatic $^{25}$ women. Thus, Carter et $\mathrm{al}^{17}$ developed and validated a multivariable prediction model (QUiPP app) in women with symptoms of PTL showing similar diagnostic performance to our prediction model with the strength to be a minimally invasive tool. However, what is of note in Carter et $\mathrm{al}^{17}$ paper is the low prevalence of spontaneous delivery within 7 days reported in their validation cohort $(4.9 \%$ vs $57 \%$ observed in our cohort).

Based on these differences, we might hypothesize that QUiPP app was used by the authors as a screening tool to increase confidence in the admission decision of women attending with symptoms. In our admission decision, we are already performing a QUiPP-like screening because we take into consideration gestational age and cervical length. This explains the high rate of spontaneous delivery within 7 days observed in our validation cohort. What differentiates our model from QUiPP app is that our model was developed not to be a screening tool but to be a diagnostic tool of spontaneous delivery within 7 days.

Our model integrates not only maternal (gestational age) and US factors (cervical length measurement), as QUiPP app does, but also information related to IAI (amniotic fluid glucose and IL-6). To include information related to IAI in a prediction model of spontaneous delivery within 7 days leads to a better prognosis and more efficient clinical management. Up to $40 \%$ of women with early diagnosis of PTL (< 28 weeks) have IAI (with or without $\mathrm{MIAC}^{2-4}$ ). In addition, early spontaneous preterm delivery ${ }^{2}$ is considered to most likely be related to this 
TABLE 2 Multivariable prediction models of MIAC and spontaneous delivery within 7 days after admission using stepwise
logistic regression analysis

\begin{tabular}{|c|c|c|c|c|c|c|c|}
\hline \multirow[b]{2}{*}{ Variables } & \multicolumn{2}{|c|}{$\begin{array}{l}\text { Spontaneous delivery } \\
\text { within } 7 \text { days }\end{array}$} & \multirow[b]{2}{*}{$P$ value } & \multirow[b]{2}{*}{ Variables } & \multicolumn{2}{|l|}{ MIAC } & \multirow[b]{2}{*}{$P$ value } \\
\hline & $\mathrm{OR}$ & $95 \% \mathrm{Cl}$ & & & $\mathrm{OR}$ & $95 \% \mathrm{Cl}$ & \\
\hline GA at amniocentesis, wks & 1.141 & $1.007-1.292$ & .038 & $\mathrm{CRP}(\mathrm{mg} / \mathrm{L})$ & 1.184 & $1.017-1.380$ & .030 \\
\hline Cervical length, mm & 0.951 & $0.913-0.990$ & .014 & AF glucose (mg/dL) & 0.854 & $0.816-0.893$ & $<.001$ \\
\hline AF glucose, mg/dL & 0.947 & $0.921-0.973$ & $<.001$ & & & & \\
\hline AF IL-6 (log) & 4.211 & $2.316-7.657$ & $<.001$ & & & & \\
\hline
\end{tabular}

$A F$, amniotic fluid; $C l$, confidence interval; $C R P$, C-reactive protein; $L L-6$, interleukin-6; $M I A C$, microbial invasion of the amniotic cavity; $O R$, odds ratio.

Cobo et al. Prediction models of spontaneous preterm delivery and microbial invasion of the amniotic cavity in preterm labor. Am J Obstet Gynecol 2020.

inflammatory condition. Finally, there has been widely reported the association of IAI with a worse perinatal outcome. ${ }^{3,4,26}$

There have been other authors who proposed a prediction model of spontaneous preterm delivery including IAI information. Thus, Holst et $\mathrm{al}^{27}$ proposed a good model to predict spontaneous delivery within 7 days including a combination of amniotic fluid and cervical fluid inflammatory proteins using multiplexed immunoassay technology. However, these results were not validated in an independent cohort, thus limiting the reproducibility of their findings. Similarly, Hitti et $\mathrm{al}^{19}$ and
Combs et $\mathrm{al}^{18}$ developed and validated 2 different predictive models of MIAC in women with PTL including different proteins measured in the cervicovaginal fluid. The main limitation is that such protein measures are not readily available in clinical laboratories.

Conversely, we propose clinically feasible prediction models that can be used in clinical decision making. Indeed, all the variables included in the models reported here can be measured immediately after admission (such as gestational age, US cervical length) or within a few hours (eg, maternal CRP, amniotic fluid glucose). There are currently even IL-6 bedside tests ${ }^{13-15}$ that provide rapid information and show a good correlation with the gold standard enzymelinked immunoassay analysis.

\section{Clinical implications}

For a clinical perspective, the classification of the risk of spontaneous delivery within 7 days and/or MIAC into low and high risk might help clinicians to more efficiently manage women with diagnosis of PTL. In the high-risk group, expected early delivery should be planned with antenatal strategies that have shown to improve neonatal outcome, such as patient transfer to facilities with neonatal intensive care units, treatment with antenatal steroids $^{28}$ and

\section{TABLE 3} Diagnostic performance of MIAC and spontaneous delivery within 7 days after admission in the derivation and the
validation cohorts

\begin{tabular}{|c|c|c|c|c|}
\hline \multirow[b]{2}{*}{ Variables } & \multicolumn{2}{|c|}{ Spontaneous delivery within 7 days } & \multicolumn{2}{|l|}{ MIAC } \\
\hline & $\begin{array}{l}\text { Derivation } \\
\text { cohort }(n=201)\end{array}$ & $\begin{array}{l}\text { Validation } \\
\text { cohort }(n=67)\end{array}$ & $\begin{array}{l}\text { Derivation } \\
\text { cohort }(n=263)\end{array}$ & $\begin{array}{l}\text { Validation } \\
\text { cohort }(n=95)\end{array}$ \\
\hline AUC $(95 \% \mathrm{Cl})$ & $0.88(0.83-0.93)$ & $0.86(0.77-0.95)$ & $0.94(0.89-0.98)$ & $0.83(0.70-0.96)$ \\
\hline Sensitivity n (\%) & $55 / 67$ (82.09) & 33/38 (86.84) & $39 / 47$ (82.98) & $16 / 21(76.19)$ \\
\hline Specificity n (\%) & $114 / 134(85.07)$ & $20 / 29(68.97)$ & 207/216 (95.83) & 68/74 (91.89) \\
\hline Positive predictive value $\mathrm{n}(\%)$ & $55 / 75(73.3)$ & $33 / 42(78.57)$ & $39 / 48(81.25)$ & $16 / 22(72.73)$ \\
\hline Negative predictive value $\mathrm{n}(\%)$ & $114 / 126(90.48)$ & $20 / 25(80)$ & 207/215 (96.28) & 68/73 (93.15) \\
\hline Positive LR & 4.53 & 2.80 & 19.91 & 9.40 \\
\hline Negative LR & 0.1838 & 0.1908 & 0.1776 & 0.2591 \\
\hline
\end{tabular}

$A U C$, area under curve; $C l$, confidence interval; $L R$, likelihood ratio; MIAC: microbial invasion of the amniotic cavity.

Cobo et al. Prediction models of spontaneous preterm delivery and microbial invasion of the amniotic cavity in preterm labor. Am J Obstet Gynecol 2020. 


\section{FIGURE 2}

\section{Fagan nomogram of the validation cohort}
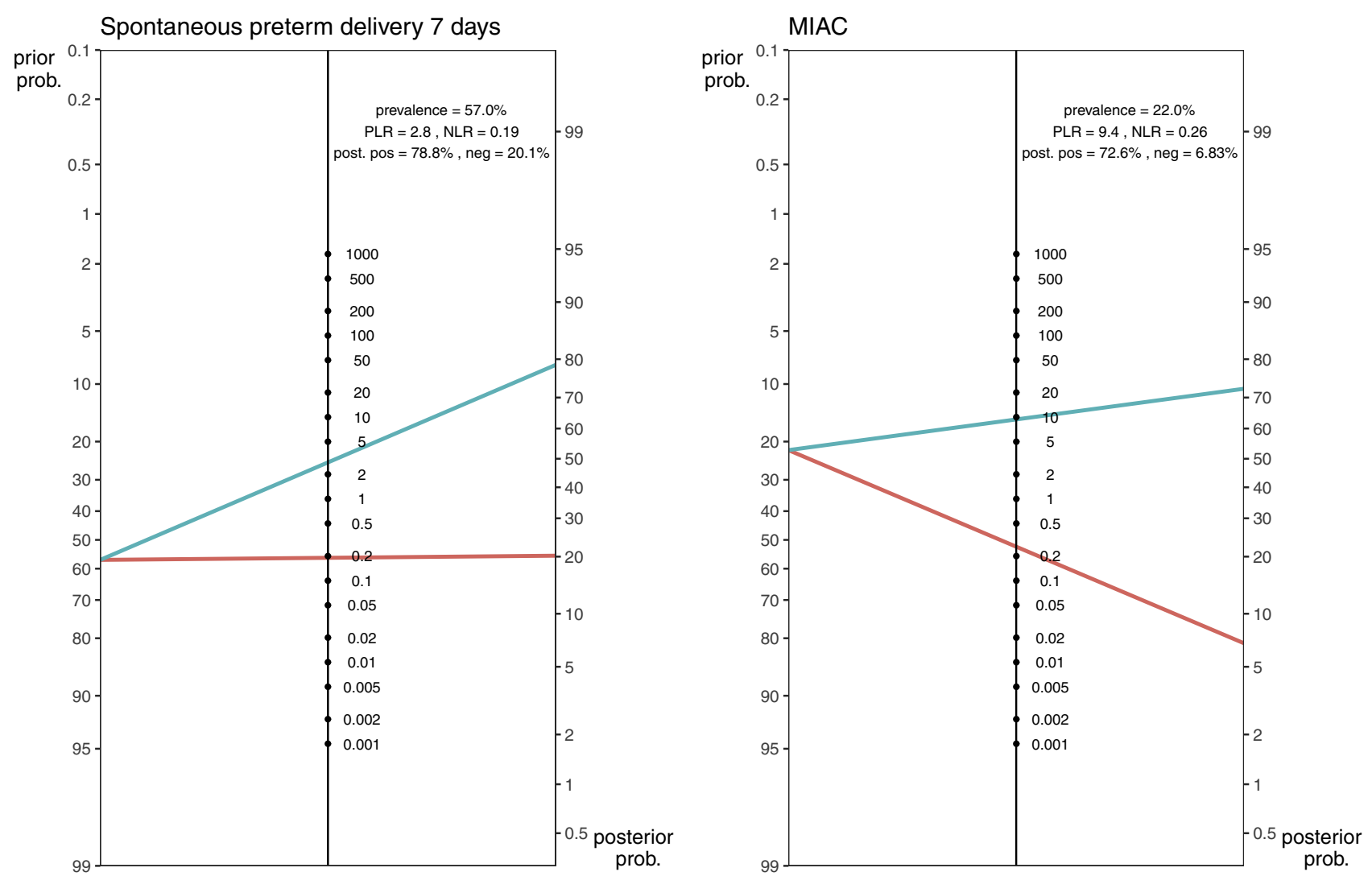

Cobo et al. Prediction models of spontaneous preterm delivery and microbial invasion of the amniotic cavity in preterm labor. Am J Obstet Gynecol 2020.

magnesium sulfate for neuroprotection, ${ }^{29}$ and, for those with MIAC, early initiation of broad-spectrum antibiotics. $^{30}$

In this regard, although systematic antenatal antibiotic prophylaxis has been questioned in women with PTL, ${ }^{31,32}$ emerging data suggest that eradication of MIAC and IAI is possible in a substantial number of women with PTL after early administration of broadspectrum antibiotic treatment. ${ }^{30}$

With respect to the low-risk group, the management of these women could be potentially ameliorated with a lowerlevel intensity intervention.

\section{Research implications}

Future studies are required to prospectively evaluate the influence of these models on improving clinical management and the potential benefit of early antibiotic treatment in women with a high-predicted risk of MIAC. Studies evaluating the cost-effectiveness of our models (eg, hospital stay length, cost of treatment, or work leave) are also warranted.

\section{Strengths and limitations}

A main strength of this study is that the model was validated in an independent sample. We included a wellcharacterized large cohort with information of MIAC and with close perinatal follow-up. Moreover, the diagnosis of MIAC was based on microbial cultures as well as PCR targeting the $16 \mathrm{~S}$ ribosomal RNA gene sequence. Moreover, all the predictors included in the 2 models are variables that can be obtained at admission or within 24 hours in most clinical settings.

Finally, we evaluated whether clinical management with tocolysis interfered with the outcome of spontaneous delivery within 7 days in some women (eg, discontinuation of tocolysis if amniotic fluid cultures were positive or glucose was $<5 \mathrm{mg} / \mathrm{dL}$ ) and found no association between them (Supplemental Table 1). This might be due to the fact that, regardless of our management, these women with MIAC will inevitably deliver in few days because of the inflammatory exposition. ${ }^{3,4,26}$

As limitations, IL-6 was measured in frozen samples, although there is strong evidence showing a good correlation between frozen and fresh samples. ${ }^{13,28}$ Finally, this study was not designed to evaluate whether our prediction models improve maternal and neonatal outcomes.

\section{Conclusion}

In conclusion, we propose 2 clinically feasible multivariable prediction models 
that may help clinicians to individualize the management of women admitted with a diagnose of PTL before 34 weeks of gestation: first, targeting the high-risk group of spontaneous delivery within 7 days after admission and/or of MIAC who require efficient planning of expected early delivery; and second, avoiding unnecessary overtreatment in the low-risk group.

\section{Acknowledgment}

This research used the Hospital Clínic-IDIBAPS Biobank.

\section{References}

1. Goldenberg RL, Culhane JF, lams JD, Romero R. Epidemiology and causes of preterm birth. Lancet 2008;371:75-84.

2. Palacio M, Cobo T, Bosch J, et al. Cervical length and gestational age at admission as predictors of intra-amniotic inflammation in preterm labor with intact membranes. Ultrasound Obstet Gynecol 2009;34:441-7.

3. Combs CA, Gravett M, Garite TJ, et al. Amniotic fluid infection, inflammation, and colonization in preterm labor with intact membranes. Am J Obstet Gynecol 2014;210:125.e1-5.

4. Romero R, Miranda J, Chaiworapongsa T, et al. Prevalence and clinical significance of sterile intra-amniotic inflammation in patients with preterm labor and intact membranes. Am J Reprod Immunol 2014;72:458-74.

5. Berghella V, Palacio M, Ness A, Alfirevic Z, Nicolaides $\mathrm{KH}$, Saccone G. Cervical length screening for prevention of preterm birth in singleton pregnancy with threatened preterm labor: systematic review and meta-analysis of randomized controlled trials using individual patient-level data. Ultrasound Obstet Gynecol 2017;49:322-9.

6. Berghella V, Saccone G. Fetal fibronectin testing for prevention of preterm birth in singleton pregnancies with threatened preterm labor: a systematic review and metaanalysis of randomized controlled trials. Am J Obstet Gynecol 2016;215:431-8.

7. Esplin MS, Elovitz MA, lams JD, et al. Predictive accuracy of serial transvaginal cervical lengths and quantitative vaginal fetal fibronectin levels for spontaneous preterm birth among nulliparous women. JAMA 2017;317:1047-56.

8. Lisonkova S, SabrY, Joseph KS. Diagnosis of subclinical amniotic fluid infection prior to rescue cerclage using gram stain and glucose tests: an individual patient meta-analysis. J Obstet Gynaecol Canada 2014;36:116-22.

9. Garry D, Figueroa R, Aguero-Rosenfeld M, Martinez E, Visintainer P, Tejani N. A comparison of rapid amniotic fluid markers in the prediction of microbial invasion of the uterine cavity and preterm delivery. Am J Obstet Gynecol 1996;175:1336-41.

10. Gauthier DW, Meyer WJ, Bieniarz A. Correlation of amniotic fluid glucose concentration and intraamniotic infection in patients with preterm labor or premature rupture of membranes. Am J Obstet Gynecol 1991;165(4 Pt 1): 1105-10.

11. Greig PC, Ernest JM, Teot L. Low amniotic fluid glucose levels are a specific but not a sensitive marker for subclinical intrauterine infections in patients in preterm labor with intact membranes. Am J Obstet Gynecol 1994;171: 365-70; discussion 370-1.

12. Romero R, Jimenez $C$, Lohda AK, et al. Amniotic fluid glucose concentration: a rapid and simple method for the detection of intraamniotic infection in preterm labor. Am J Obstet Gynecol 1990;163:968-74.

13. Chaemsaithong $P, \quad$ Romero $R$, Korzeniewski SJ, et al. A point of care test for interleukin-6 in amniotic fluid in preterm prelabor rupture of membranes: a step toward the early treatment of acute intra-amniotic inflammation/ infection. J Matern Fetal Neonat Med 2015: $1-8$.

14. Chaemsaithong $P$, Romero R, Korzeniewski SJ, et al. A rapid interleukin-6 bedside test for the identification of intraamniotic inflammation in preterm labor with intact membranes. J Matern Fetal Neonat Med 2015:1-11.

15. Kacerovsky M, Musilova I, Hornychova H, et al. Bedside assessment of amniotic fluid interleukin-6 in preterm prelabor rupture of membranes. Am J Obstet Gynecol 2014;211: 385.e1-9.

16. Rolnik DL, Wright D, Poon LCY, et al. ASPRE trial: performance of screening for preterm preeclampsia. Ultrasound Obstet Gynecol 2017; 50:492-5.

17. Carter J, Seed PT, Watson HA, et al. Development and validation of predictive models for QUiPP App v.2: tool for predicting preterm birth in women with symptoms of threatened preterm labor. Ultrasound Obstet Gynecol 2020;55:357-67.

18. Combs CA, Garite TJ, Lapidus JA, et al. Detection of microbial invasion of the amniotic cavity by analysis of cervicovaginal proteins in women with preterm labor and intact membranes. Am J Obstet Gynecol 2015;212:482. e1-12.

19. Hitti J, Lapidus JA, Lu X, et al. Noninvasive diagnosis of intraamniotic infection: proteomic biomarkers in vaginal fluid. Am J Obstet Gynecol 2010;203:32.e1-8.

20. Holst RM, Hagberg $H$, Wennerholm UB, Skogstrand K, Thorsen P, Jacobsson B. Prediction of microbial invasion of the amniotic cavity in women with preterm labour: analysis of multiple proteins in amniotic and cervical fluids. BJOG 2011;118:240-9.

21. Gibbs RS, Blanco JD, St Clair PJ, Castaneda YS. Quantitative bacteriology of amniotic fluid from women with clinical intraamniotic infection at term. J Infect Dis 1982;145:1-8.

22. Robinson HP. Sonar measurement of fetal crown-rump length as means of assessing maturity in first trimester of pregnancy. Br Med $\mathrm{J}$ 1973;4:28-31.

23. Peduzzi P, Concato J, Kemper E, Holford TR, Feinstein AR. A simulation study of the number of events per variable in logistic regression analysis. J Clin Epidemiol 1996;49: 1373-9.

24. Musilova I, Bestvina T, Stranik J, Stepan M, Jacobsson B, Kacerovsky M. Transabdominal amniocentesis is a feasible and safe procedure in preterm prelabor rupture of membranes. Fetal Diagn Ther 2017:42:257-61.

25. Celik E, To M, Gajewska K, Smith GC, Nicolaides $\mathrm{KH}$. Fetal Medicine Foundation Second Trimester Screening Group. Cervical length and obstetric history predict spontaneous preterm birth: development and validation of a model to provide individualized risk assessment. Ultrasound Obstet Gyneco 2008;31:549-54.

26. Cobo T, Vives I, Rodriguez-Trujillo A, et al. Impact of microbial invasion of amniotic cavity and the type of microorganisms on short-term neonatal outcome in women with preterm labor and intact membranes. Acta Obstet Gynecol Scand 2017; 96:570-9.

27. Holst RM, Hagberg $H$, Wennerholm UB, Skogstrand K, Thorsen P, Jacobsson B. Prediction of spontaneous preterm delivery in women with preterm labor: analysis of multiple proteins in amniotic and cervical fluids. Obstet Gynecol 2009;114(2 Pt 1):268-77.

28. Roberts D, Brown J, Medley N, Dalziel SR. Antenatal corticosteroids for accelerating fetal lung maturation for women at risk of preterm birth. Cochrane Database Syst Rev 2017;3: CD004454.

29. Chollat C, Marret S. Magnesium sulfate and fetal neuroprotection: overview of clinical evidence. Neural Regen Res 2018;13:2044-9.

30. Yoon BH, Romero R, Park JY, et al. Antibiotic administration can eradicate intraamniotic infection or intra-amniotic inflammation in a subset of patients with preterm labor and intact membranes. Am J Obstet Gynecol 2019;221:142.e1-22.

31. Kenyon SL, Taylor DJ, Tarnow-Mordi W. Broad-spectrum antibiotics for spontaneous preterm labour: the ORACLE II randomised trial. ORACLE Collaborative Group. Lancet 2001;357:989-94.

32. Kenyon S, Pike K, Jones DR, et al. Childhood outcomes after prescription of antibiotics to pregnant women with spontaneous preterm labour: 7-year follow-up of the ORACLE II trial. Lancet 2008;372:1319-27.

\section{Author and article information}

From the BCNatal-Barcelona Center for Maternal-Fetal and Neonatal Medicine (Hospital Clínic and Hospital Sant 
Joan de Deu), Institut Clínic de Ginecología, Obstetrícia I Neonatología, Fetal i+D Fetal Medicine Research Center, Institut d'Investigacions Biomèdiques August Pi I Sunyer, University of Barcelona (Drs Cobo, Aldecoa, Figueras, Herranz, Ferrero, Izquierdo, Murillo, Amoedo, Rueda, Martínez-Portilla, Gratacós, and Palacio), the Centre for Biomedical Research on Rare Diseases (Drs Cobo, Figueras, Gratacós, and Palacio), and the Microbiology, Biomedical Diagnostic Center, Hospital Clínic and ISGlobal (Barcelona Institute for Global Health), University of Barcelona (Dr Bosch), Barcelona, Spain.
${ }^{1}$ These authors contributed equally as last authors of the paper.

Received Dec. 17, 2019; revised Jan. 29, 2020; accepted Feb. 27, 2020.

This project has been partially funded with support of the Instituto de Salud Carlos III (PI15/00344, Pl17/ 00675) integrado en el Plan Nacional de I+D+I y cofinanciado por el ISCIII-Subdirección General de Evaluación y el Fondo Europeo de Desarrollo Regional "Una Manera de Hacer Europa" and the Cellex Foundation. T.C. has received funding from the
Departament de Salut under grant SLT008/18/00126. Additionally, E.G. and F.C. have received funding form the "La Caixa" Foundation under grant agreements LCF/PR/GN14/10270005 and LCF/PR/GN18/ 10310003, Cerebra Foundation for the Brain Injured Child (Carmarthen, Wales, United Kingdom) and AGAUR 2017 SGR grant 1531 is funded by Generalitat Catalunya, Catalonia.

The authors report no conflict of interest.

Corresponding author: Teresa Cobo, MD, PhD. tcobo@clinic.cat 


\section{SUPPLEMENTAL TABLE 1 \\ Information about microorganisms isolated in the amniotic fluid}

\section{Variables}

1

2

3

4

5

6

7

8

9

10

11

12

13

14

15

16

17

18

19

20

21

22

23

24

25

26

27

28

29

30

31

32

33

34

35

36

$\frac{36}{37}$

37

38

39
Microorganisms in the amniotic fluid

Ureaplasma spp

Ureaplasma spp

Ureaplasma spp

Ureaplasma spp

Ureaplasma spp

Ureaplasma spp

Ureaplasma spp

Ureaplasma spp

Ureaplasma spp

Ureaplasma spp

Ureaplasma spp

Ureaplasma spp

Ureaplasma spp

Ureaplasma sp., Streptococcus anginosus

Ureaplasma spp, Fusobacterium spp

Ureaplasma spp

Ureaplasma spp

Ureaplasma spp

Ureaplasma spp

Ureaplasma spp

Ureaplasma spp

Ureaplasma spp

Ureaplasma spp

Ureaplasma spp

Ureaplasma spp

Ureaplasma spp

Ureaplasma spp

Ureaplasma spp, Haemophilus influenzae

Ureaplasma spp., Mycoplasma hominis

Ureaplasma spp, Fusobacterium spp, Candida albicans

Ureaplasma spp

Mycoplasma hominis

Mycoplasma hominis, Fusobacterium spp

Fusobacterium spp

Fusobacterium nucleatum

Fusobacterium spp

Fusobacterium spp

Fusobacterium nucleatum

Fusobacterium nucleatum
AF IL-6, ng/mL

0.102

20.106

90.832

892.642

22.891

1.799

133.250

158.600

37.760

4.306

9.005

0.376

3.736

161.051

24.522

236.856

80.7325

75.7254

838.025

148.620

151.632

125.220

26.7074

48.1035

139.401

152.103

1214.000

207.199

340.687

24.432

41.151

598.0

30.313

2.774

1888.000

2.790

44.420

591.869

789.989
Latency to delivery, $\mathrm{d}$

3.00

15.00

1.00

15.00

1.00

6.00

1.00

1.00

1.00

3.00

1.00

13.00

2.00

1.00

0.00

1.00

12.00

1.00

4.00

0.00

3.00

1.00

4.00

2.00

3.00

3.00

3.00

.00

1.00

2.00

36.00

0.00

2.00

0.00

17.00

0.00

3.00

1.00

0.00

Cobo et al. Prediction models of spontaneous preterm delivery and microbial invasion of the amniotic cavity in preterm labor. Am J Obstet Gynecol 2020.

(continued) 


\begin{tabular}{|c|c|c|c|}
\hline Variables & Microorganisms in the amniotic fluid & AF IL-6, ng/mL & Latency to delivery, $\mathrm{d}$ \\
\hline 40 & Fusobacterium nucleatum & 1595.000 & 2.00 \\
\hline 41 & Fusobacterium spp & 498.415 & 0.00 \\
\hline 42 & Fusobacterium nucleatum & 837.164 & 5.00 \\
\hline 43 & Fusobacterium nucleatum, Streptococcus viridans & 2879.000 & 0.00 \\
\hline 44 & Fusobacterium spp, Candida albicans & 1332.000 & 1.00 \\
\hline 45 & Fusobacterium nucleatum, Candida albicans & 0.339 & 1.00 \\
\hline 46 & Streptococcus viridans & 2.829 & 1.00 \\
\hline 47 & Streptococcus agalactiae, Candida albicans & 2.460 & 2.00 \\
\hline 48 & Streptococcus mitis & 1.176 & 53.00 \\
\hline 49 & Streptococcus pyogenes & 0.2627 & 46.00 \\
\hline 50 & Streptococcus agalactiae & 542.438 & 0.00 \\
\hline 51 & Listeria monocytogenes & 242.202 & 3.00 \\
\hline 52 & Listeria monocytogenes & 18.151 & 0.00 \\
\hline 53 & Listeria monocytogenes & 127.575 & 0.00 \\
\hline 54 & Lactobacillus & 28.060 & 11.00 \\
\hline 55 & Lactobacillus & 118.842 & 1.00 \\
\hline 56 & Escherichia coli & 281.509 & 0.00 \\
\hline 57 & Escherichia coli & 2.8948 & 0.00 \\
\hline 58 & Escherichia coli & 20.8493 & 0.00 \\
\hline 59 & Capnocytophaga sputigena & 38.480 & 1.00 \\
\hline 60 & Capnocytophaga sputigena & 123.386 & 0.00 \\
\hline 61 & Candida albicans & 142.649 & 0.00 \\
\hline 62 & Candida albicans & 6.020 & 2.00 \\
\hline 63 & Candida albicans & 14.720 & 7.00 \\
\hline 64 & Leptotrichia spp & 262.367 & 1.00 \\
\hline 65 & Proteus mirabilis & 48.940 & 1.00 \\
\hline 66 & Prevotella amnii & 413.000 & 1.00 \\
\hline 67 & Peptostreptococcus & 36.499 & 0.00 \\
\hline 68 & Bacteroides fragilis & 696.715 & 0.00 \\
\hline
\end{tabular}




\section{SUPPLEMENTAL TABLE 2 \\ Maternal characteristics and perinatal outcomes according to the outcome of MIAC}

\begin{tabular}{|c|c|c|c|}
\hline Variables & $\operatorname{MIAC}(n=68)$ & No MIAC $(\mathrm{n}=290)$ & $P$ value \\
\hline Maternal age, y & $33.3(26.3,36.5)$ & $31.8(27.9,36.3)$ & 233 \\
\hline White ethnicity & $47(69)$ & $202(70)$ & .541 \\
\hline Smoking & 8/59 (14) & $35 / 239(15)$ & 1.000 \\
\hline Nulliparity & $35(52)$ & $146(50)$ & .893 \\
\hline Prior preterm birth & $6(9)$ & $31(11)$ & .825 \\
\hline Cervical length, mm & $5(0,14)$ & $11(5 ; 18)$ & .002 \\
\hline CRP, mg/L & $3.4(1.1,7.3)$ & $0.6(0.3,1.4)$ & $<.001$ \\
\hline WBC $\left(\times 10^{9} / \mathrm{L}\right)$ & $14,110(12,085,1,7285)$ & $12,090(9890,14,720)$ & $<.001$ \\
\hline GA at admission, wks & $26.4(24.8,30.5)$ & $28.6(25.7,31.0)$ & .017 \\
\hline GA at amniocentesis, wks & $26.4(24,8,30.5)$ & $28.6(25.7,31.0)$ & .018 \\
\hline AF glucose, mg/dL & $4(0,6)$ & $33(24,44)$ & $<.001$ \\
\hline AF IL-6, ng/mL & $48.1(10.4,273.9)$ & $2.2(0.9,10.6)$ & $<.001$ \\
\hline Antenatal steroids & $57 / 59(97)$ & 260/283 (92) & .276 \\
\hline Antenatal antibiotics & $66(97)$ & 145/289 (50) & $<.001$ \\
\hline GA at delivery, wks & $26.9(25.2,31.1)$ & $35.0(29.7,38.3)$ & $<.001$ \\
\hline Spontaneous onset of labor & $49(72)$ & $219(76)$ & .539 \\
\hline Latency to delivery, $d$ & $1(0,3)$ & $31(6,62)$ & $<.001$ \\
\hline Clinical chorioamnionitis at delivery & $39 / 66$ (59) & $27 / 283(10)$ & $<.001$ \\
\hline
\end{tabular}

Continuous variables were compared using a nonparametric Mann-Whitney $U$ test presented as medians (25th and 75 th interquartile percentiles). Categorical variables were compared using $\chi^{2}$ or Fisher exact tests and presented as number (percentage).

$A F$, amniotic fluid; CRP, C-reactive protein; GA, gestational age; MIAC, microbial invasion of the amniotic cavity; WBC, white blood cells.

Cobo et al. Prediction models of spontaneous preterm delivery and microbial invasion of the amniotic cavity in preterm labor. Am J Obstet Gynecol 2020. 


\section{SUPPLEMENTAL TABLE 3 \\ Maternal characteristics and perinatal outcomes according to the outcome of sPTD within 7 days}

\begin{tabular}{lccr} 
Variables & sPTD, $7 \mathrm{~d}(\mathbf{n}=105)$ & No sPTD, $7 \mathrm{~d}(\mathbf{n}=163)$ & $P$ value \\
\hline Maternal age, $y$ & $33.0(27.6,36.8)$ & $31.9(27.6,36.3)$ & .554 \\
\hline White ethnicity & $70(67)$ & $121(74)$ & .167 \\
\hline Smoking & $21 / 98(21)$ & $10 / 126(8)$ & .006 \\
\hline Nulliparity & $58(55)$ & $80(49)$ & .381 \\
\hline Prior preterm birth & $12(11)$ & $18(11)$ & 1.000 \\
\hline Cervical length, mm & $6(0,16)$ & $11(6,18)$ & $<.001$ \\
\hline CRP, mg/L & $1.8(0.7,4.4)$ & $0.5(0.3,1.1)$ & $<.001$ \\
\hline WBC $\left(\times 10^{9} / \mathrm{L}\right.$ & $14040(11875,17340)$ & $11540(9700,14080)$ & $<.001$ \\
\hline GA at admission, wks & $27.4(25.0,31.0)$ & $28.9(26.0,31.0)$ & .148 \\
\hline GA at amniocentesis, wks & $27.4(25.0,31.0)$ & $29.0(26.0,31.0)$ & .171 \\
\hline AF glucose, mg/dL & $16(3.5,30)$ & $36(26,46)$ & $<.001$ \\
\hline AF IL-6, ng/mL & $32.0(4.5,127.6)$ & $1.3(0.8,2.89)$ & $<.001$ \\
\hline Antenatal steroids & $97 / 99(98)$ & $147 / 160(92)$ & .054 \\
\hline Antenatal antibiotics & $95(91)$ & $66 / 162(41)$ & $<.001$ \\
\hline GA at delivery, wks & $27.4(25.1,31.3)$ & $37.3(33.0,38.9)$ & $<.001$ \\
\hline Spontaneous onset of labor & $105(100)$ & $163(100)$ & - \\
\hline Latency to delivery, d & $2(1,3)$ & $49(27,72)$ & $<.001$ \\
\hline Clinical chorioamnionitis at delivery & $37 / 99(37)$ & $10 / 162(6)$ & $<.001$
\end{tabular}

Continuous variables were compared using a nonparametric Mann-Whitney $U$ test presented as medians (25th and 75 th interquartile percentiles). Categorical variables were compared using $\chi^{2}$ or Fisher exact tests and presented as number (percentage).

$A F$, amniotic fluid; CRP, C-reactive protein; GA, gestational age; MIAC, microbial invasion of the amniotic cavity; WBC, white blood cells.

Cobo et al. Prediction models of spontaneous preterm delivery and microbial invasion of the amniotic cavity in preterm labor. Am J Obstet Gynecol 2020. 


\section{SUPPLEMENTAL TABLE 4}

Association between model variables for prediction of spontaneous preterm delivery at 7 days after nearest neighbor propensity score matching

\begin{tabular}{lllll} 
Outcome & GA at admission & Cervical length & AF glucose & AF IL-6 \\
\hline sPTD within 7 days & Coefficient $(95 \%$ Cl$)$ & & & Reference \\
\hline No tocolysis $(n=10)$ & Reference & Reference & Reference & $-0.49(-0.13$ to 0.31$)$
\end{tabular}

Estimated average treatment effect in population comparing tocolysis vs. no tocolysis

\begin{tabular}{llllll}
\hline Outcome & Coefficient & SE & $95 \% \mathrm{Cl}$ & $P$ value \\
\hline sPTD within 7 days & 0.22 & 0.2 & -0.18 to 0.62 & 0.285 \\
\hline
\end{tabular}

$A F$, amniotic fluid; $C l$, confidence interval; $G A$, gestational age; $I L$, interleukin; $s P T D$, spontaneous preterm delivery.

Cobo et al. Prediction models of spontaneous preterm delivery and microbial invasion of the amniotic cavity in preterm labor. Am J Obstet Gynecol 2020. 